\title{
STUDYING OF THE CONTACT ANGLE HYSTERESIS ON VARIOUS SURFACES
}

\author{
E. O. Kirichenko ${ }^{1,2}$, and E. Ya. Gatapova ${ }^{1, *}$ \\ ${ }^{1}$ Kutateladze Institute of Thermophysics SB RAS, 630090 Novosibirsk, Russia \\ ${ }^{2}$ Novosibirsk State University (NSU), 630090 Novosibirsk, Russia
}

\begin{abstract}
This paper is devoted to investigation of the contact angle hysteresis on various surfaces. It was carried out by two different methods: measuring the advancing and the receding contact angles and measuring the contact angles at water droplet evaporation under isothermal conditions. Data obtained using two methods have been compared. The influence of the contact angle hysteresis on the mode of the drop evaporation has been shown.
\end{abstract}

\section{Introduction}

In connection with rapid development of electronic technology there is a need in creation of new highly efficient methods of heat transfer enhancement [1]. Significant improvement in the performance of heat exchange devices may be reached by using surfaces with nanocoating [2]. Studies of the properties of these surfaces, such as the contact angle hysteresis $[3,4]$ play an important role in the choice of their application $[5,6]$.

In this paper, we study eight identical glass substrates with coatings applied using different technologies. The following coatings were considered: substrate manufactured using the silanization technology (absorption of trichloroctadecylsilane by silicon oxide); applying the photoresists of different types on a substrate, and applying a Teflon layer at various speeds of rotation. The resulting surfaces have different properties.

On these substrates, the contact angle and the contact angle hysteresis were measured by different methods. The measurements were carried out using the Drop Shape Analyzer from Kruss.

\section{Experimental setup and research methods}

The Drop Shape Analyzer (Kruss company) allows measuring the contact angle by the sessile method or the captive bubble method. The measuring range is $1-180^{\circ}$, and the resolution is $\pm 0.1^{\circ}$. For the analysis of the drop shape 6 methods are used (polynomial, length-height, the conical segment, the circumference, Yuong-Laplace equation, and the distance between the points). The liquid is dosed automatically. High-precision systems for dosing and positioning the liquid droplets in combination with the system of recording,

* Corresponding author: egatapova@gmail.com 
analysis of the video (images) and calculation of surface energy and surface tension provide for measurements in an automatic mode. Digital camera has recording speed of 61 - 311 frame/sec, and $\mathrm{max} / \mathrm{min}$ frames resolution: $780 \times 580 / 780 \times 60 \mathrm{px}$. Optical system has sevenfold automatic zoom and coverage $3.7 \times 2.7-23.2 \times 17.2 \mathrm{~mm}$. Photo of the experimental setup is presented in figure 1 .

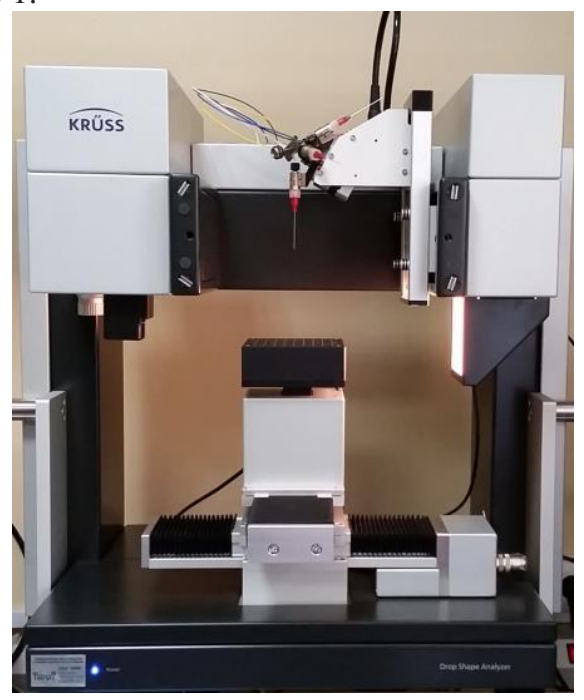

Fig. 1. A device for measuring the contact angle DSA100-E KRUSS.

The contact angle hysteresis was studied by two methods. The first method is measuring the advancing and the receding contact angles. The working fluid is ultraclean deionized water (MilliQ). Using the syringe pump, a drop is dispensed on the surface with constant rate of $0.2 \mathrm{ml} / \mathrm{min}$, and at this time the advancing contact angle is determined. Then, at reaching the maximum volume of $5 \mu 1$, the syringe pump intakes the liquid with a constant rate of $0.2 \mathrm{ml} / \mathrm{min}$. At that time the receding contact angle is measured. The drop volume and the liquid flow rate are chosen based on the properties of the liquid. The difference between the values of the advancing and receding angles is the hysteresis. The second method of studying the hysteresis of the angle is isothermal evaporation. A drop of $5 \mu 1$ volume gets down to the surface and evaporates into the atmosphere. The contact angle is measured in the course of time.

\section{The results of research}

\subsection{The method of advancing and receding contact angle}

It is experimentally shown that the contact angle and the hysteresis are dependent on the technology of coating application. Figure 2 shows the dependence of the advancing/receding contact angle on the speed of the contact line on the surface, obtained by silanization. On the left, there are values of the receding contact angle, and on the right, values of the advancing contact angle. The hysteresis of the contact angle is determined in the vicinity of zero velocity of the contact line, and on this surface it is $10^{\circ}-20^{\circ}$. 


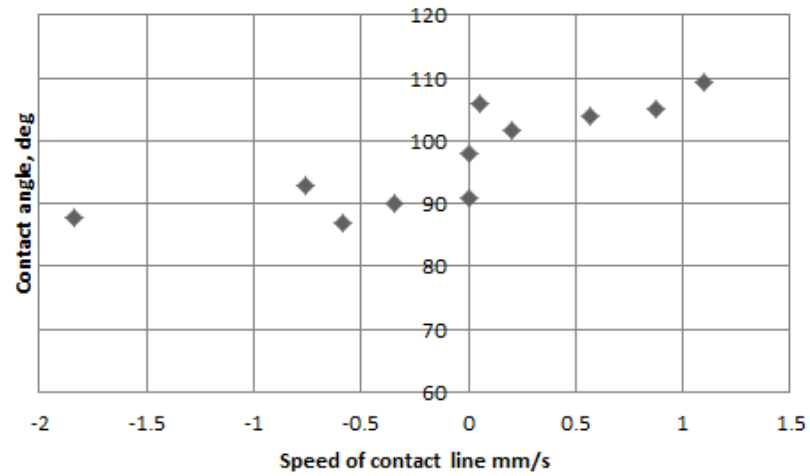

Fig. 2. The dependence of the advancing/receding contact angle on the speed of the contact line. The surface is Silane OTS.

Figure 3 shows the dependence of the advancing/receding contact angle on the speed of the contact line on the surface, obtained by application of photoresist Noa65. The value of contact angle hysteresis is about $30^{\circ}-40^{\circ}$. There is no negative velocity, since during the liquid intake the contact line remains fixed and does not move throughout the process.

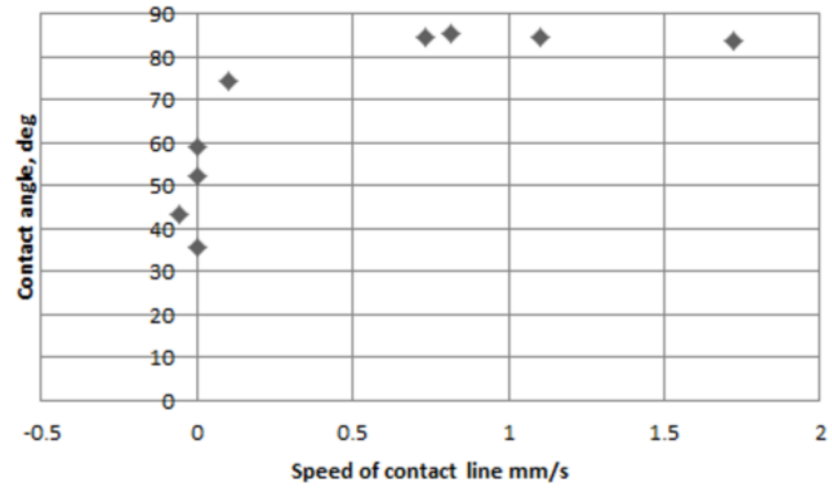

Fig. 3. The dependence of advancing/receding contact angle on the speed of the contact line. The surface is Noa65.

Fig. $4 \mathrm{a}$ and $4 \mathrm{~b}$ present typical photographs of contact angle measurements using DSA 100 of the resting water droplet of $5 \mu 1$ volume. The contact angle on the surface of the Silane OTS is $97^{\circ}$ (fig. $4 \mathrm{a}$ ), and on the surface of Noa65, it is $81^{\circ}$ (fig. $4 \mathrm{~b}$ ).

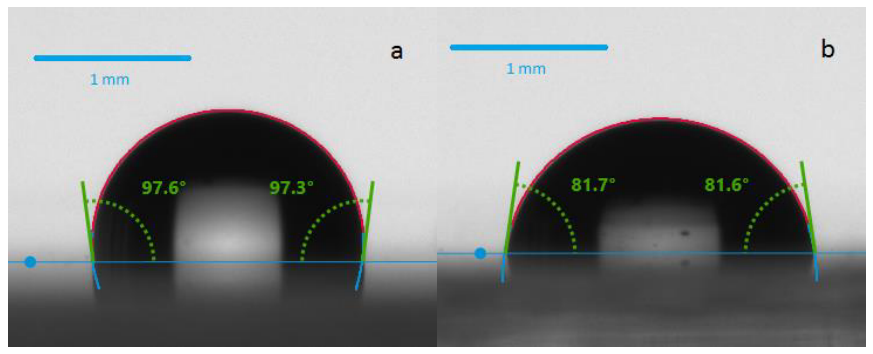

Fig. 4. Contact angle (a) OTS Silane surface b) Noa65 surface. 


\subsection{The method of isothermal droplet evaporation}

Using the isothermal evaporation method the drops with an initial volume of $5 \mu$ were investigated on the earlier described surfaces. While evaporating a liquid drop demonstrates one of three modes: the contact line moves, and the contact angle remains constant throughout the evaporation process (depinning); the contact line is fixed, and the contact angle continuously decreases (pinning); or a mixed mode. Figure 4 shows the dependence of the contact angle on the speed of the contact line on the surface of the Silane OTS.

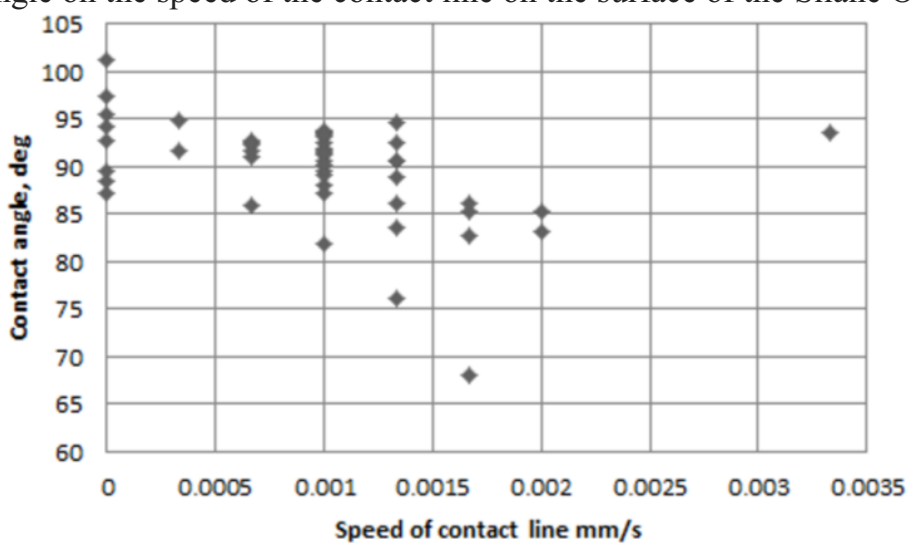

Fig. 5. The dependence of the contact angle on the speed of contact line. The surface is Silane OTS.

To compare the results with the method of advancing/receding contact angle the contact angle hysteresis is also determined in the vicinity of zero velocity of the contact line, and is $10^{\circ}-20^{\circ}$, which is consistent with the results, obtained by the first method. The average rate of change of the droplet contact radius, equal to the ratio of the initial contact radius to the drop lifetime, on the surface is $0.00085 \mathrm{~mm} / \mathrm{s}$. Relative to this speed, the speed values shown in figure 5 , are rather large.

On the Noa65 surface the hysteresis is about $50^{\circ}$ (fig. 6). Thus, the difference in determining the hysteresis by two methods is about $10^{\circ}$.

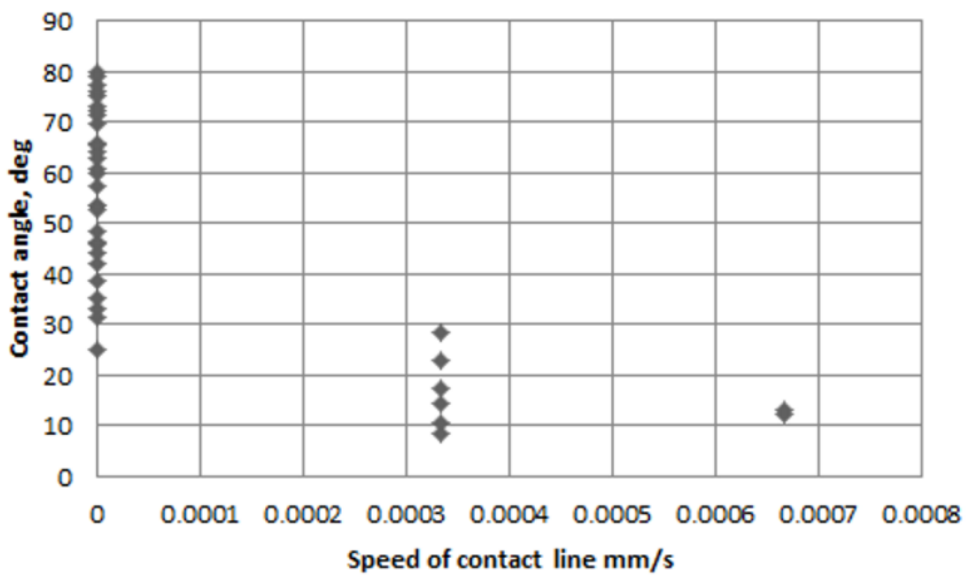

Fig. 6. The dependence of the contact angle on the speed of contact line. The surface is Noa65.

On these surfaces, the droplet demonstrates different modes of evaporation (fig. 7). On the surface obtained by silanization, the contact angle remains constant for almost all the 
time of drop evaporation. Whereas on the surface with applied photoresist, the contact line remains fixed, which leads to a permanent change in the contact angle.

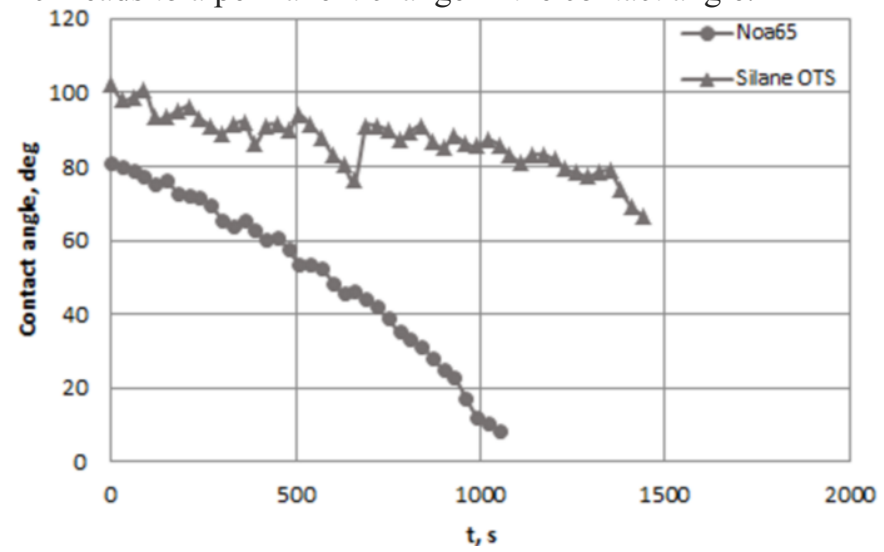

Fig. 7. The dependence of the contact angle on the time of evaporation.

The contact angle in figure 7 for both surfaces is changed in accordance with the measured the contact angle hysteresis (fig. 5, 6).

\section{Conclusions}

The investigation has been carried out to determine the contact angle hysteresis on various surfaces. The hysteresis was studied by two methods: by using standard procedures for DSA100, as well as at evaporation of water drops in the atmosphere under normal conditions. Two common types of coatings were studied: Silane and NOA 65. It has been found out that on the NOA 65 surface the difference in determining the hysteresis by two methods is about $10^{\circ}$, and on the Silane substrate the measurements coincide. The surface obtained by applying a photoresist NOA 65 can be used to create a flow with a constant contact radius. The surface obtained through silanization can be applied to organize flows with a constant contact angle.

\section{Acknowledgments}

The work was supported by the Russian Science Foundation (project No. 16-19-10675).

\section{References}

1. A. Bar-Cohen, K. Matin, N. Jankowski, D. Sharar, J. Electron. Packag. 137, 1 (2014)

2. N. Miljkovic, R. Enright, Y. Nam, K. Lopez, N. Dou, J. Sack, E.N. Wang, Nano Lett. 13, 179 (2013)

3. Jr. R.E. Johnson, R.H. Dettre, The journal of physical chemistry 68, 1 (1964)

4. O.A. Kabov, D.V. Zaitsev, Dokl. Phys. 58, 7 (2013)

5. A. Safonov, V. Sulyaeva, N. Timoshenko, E. Gatapova, O. Kabov, E. Kirichenko, A. Semenov, MATEC Web Conf. 37, Article Number 01047 (2015)

6. E.Ya. Gatapova, A.A. Semenov, D.V. Zaitsev, O.A. Kabov, Colloids Surf., A 441 (2014) 